\title{
HSP27 Inhibits Homocysteine-Induced Endothelial Apoptosis by Modulation of ROS Production and Mitochondrial Caspase-Dependent Apoptotic Pathway
}

\author{
Xin Tian, ${ }^{1}$ Lei Zhao, ${ }^{1}$ Xianjing Song, ${ }^{1}$ Youyou Yan, ${ }^{1}$ Ning Liu, ${ }^{1}$ Tianyi Li, \\ Bingdi Yan, ${ }^{2}$ and Bin Liu ${ }^{1}$ \\ ${ }^{1}$ Department of Cardiology, The Second Hospital of Jilin University, 218 Ziqiang Street, Changchun 130041, China \\ ${ }^{2}$ Department of Respiratory Medicine, The Second Hospital of Jilin University, 218 Ziqiang Street, Changchun 130041, China
}

Correspondence should be addressed to Bin Liu; guxin33@126.com

Received 13 January 2016; Revised 18 March 2016; Accepted 23 March 2016

Academic Editor: Cheng I. Cheng

Copyright (c) 2016 Xin Tian et al. This is an open access article distributed under the Creative Commons Attribution License, which permits unrestricted use, distribution, and reproduction in any medium, provided the original work is properly cited.

Objectives. Elevated plasma homocysteine (Hcy) could lead to endothelial dysfunction and is viewed as an independent risk factor for atherosclerosis. Heat shock protein 27 (HSP27), a small heat shock protein, is reported to exert protective effect against atherosclerosis. This study aims to investigate the protective effect of HSP27 against Hcy-induced endothelial cell apoptosis in human umbilical vein endothelial cells (HUVECs) and to determine the underlying mechanisms. Methods. Apoptosis, reactive oxygen species (ROS), and mitochondrial membrane potential (MMP) of normal or HSP27-overexpressing HUVECs in the presence of Hcy were analyzed by flow cytometry. The mRNA and protein expression levels were measured by quantitative real-time polymerase chain reaction (qRT-PCR) and western blot. Results. We found that Hcy could induce cell apoptosis with corresponding decrease of nitric oxide (NO) level, increase of endothelin-1 (ET-1), intracellular adhesion molecule-1 (ICAM-1), vascular cellular adhesion molecule-1 (VCAM-1), and monocyte chemoattractant protein-1 (MCP-1) levels, elevation of ROS, and dissipation of MMP. In addition, HSP27 could protect the cell against Hcy-induced apoptosis and inhibit the effect of Hcy on HUVECs. Furthermore, HSP27 could increase the ratio of Bcl-2/Bax and inhibit caspase- 3 activity. Conclusions. Therefore, we concluded that HSP27 played a protective role against Hcy-induced endothelial apoptosis through modulation of ROS production and the mitochondrial caspase-dependent apoptotic pathway.

\section{Introduction}

Atherosclerosis, a chronic immunoinflammatory disease, is the most common underlying cause of cardiovascular and peripheral vascular diseases $[1,2]$. Elevation of the plasma level of Hcy is one of the recognized independent risk factors for the development of atherosclerosis [3-5]. Evidence shows that the elevation of plasma Hcy can lead to endothelial dysfunction [6, 7], an early marker for atherosclerosis $[8,9]$. It was reported that endothelial cell apoptosis contributed to endothelial dysfunction and destabilization of atherosclerotic plaques and thrombosis [10].

HSP27, a member of the small heat shock protein family, is involved in a wide variety of cellular processes such as apoptosis [11], inflammation [12], cell migration [13], and maintenance of arterial wall homeostasis [14]. HSP27 in atherosclerotic plaques diminishes with progression of the stage of the pathology $[15,16]$ and is thereby viewed as a potential biomarker [14]. It has also been shown that HSP27 can protect cells from apoptosis induced by various stimuli in vivo and in vitro [17-20]. This cytoprotective effect of HSP27 is associated with decrease in ROS [21] and increase in glutathione [22] during oxidative stress. In addition, HSP27 acts both upstream and downstream of cytochrome $c$ release in a stimulus-dependent manner [23] and inhibits caspase- 3 activity through interaction with its inactive form, procaspase-3 [24]. However, modulation in HSP27 expression toward endothelial protection against Hcy treatment has not been investigated.

The objectives of this study were to determine the effect of HSP27 on Hcy-induced endothelial cell apoptosis and to 
elucidate the possible protective mechanism of HSP27 against endothelial dysfunction.

\section{Materials and Methods}

2.1. Materials. Rh123 mitochondrial specific fluorescent dye was purchased from Sigma. Reactive Oxygen Species Assay Kit was purchased from Beyotime Company (Shanghai, China). Dulbecco's modified Eagle's medium (DMEM) and fetal bovine serum (FBS) were purchased from GibcoInvitrogen. D,L-Hcy was purchased from Sigma (Aldrich, America). BCA Protein Assay Kit and annexin V-FITC Apoptosis Detection Kit were purchased from Keygen Company (Nanjing, China). Polyclonal antibodies against $\beta$-actin and horseradish peroxidase-conjugated secondary antibodies (goat-anti-rabbit, mouse) were purchased from Beyotime Biotechnology (Shanghai, China). Antibody for HSP27 was obtained from Abcam. Polyclonal antibodies against Bax, Bcl-2, caspase-3, and PARP were purchased from Cell Signaling Technology, Inc. (Danvers, MA, USA). Western blot detection kit was purchased from Millipore (Billerica, USA).

2.2. Cell Culture. HUVECs were maintained in DMEM supplemented with $10 \% \mathrm{FBS}$ at $37^{\circ} \mathrm{C}$ in a humidified atmosphere with $5 \% \mathrm{CO}_{2}$ and $95 \%$ air.

2.3. Measurement of HSP27 Expression in Hcy-Treated HUVECs. HUVECs were incubated in $100 \mathrm{~mm}$ plates and treated with Hcy at the concentrations of $0 \mathrm{mM}, 5 \mathrm{mM}$, and $10 \mathrm{mM}$ for $24 \mathrm{~h}$. The cells were then harvested and HSP27 expression was measured by western blot.

2.4. Cell Transfection. HUVECs were transfected with either empty pEX-4 or HSP27-pEX-4 plasmid (GenePharma, Shanghai, China). The plasmids contained green fluorescent protein (GFP) and the neomycin resistance gene. UVECs were seeded in six-well plate with $40-60 \%$ confluence $20 \mathrm{~h}$ before transfection and the plasmids were transfected by Lipofectamine ${ }^{\circledR} 2000$ (Invitrogen, Guangzhou, China) according to the user's protocol. Transfected cells were screened using $0.7 \mathrm{mg} / \mathrm{mL}$ G418 (Sigma-Aldrich, America). After 2 weeks, single G418-resistant cell clones were seeded and grown in a 96-well plate. HSP27 expression was detected using fluorescence microscopy, qRT-PCR, and western blot. HUVECs transfected with an empty plasmid were labeled as Neo while those transfected with plasmid coding HSP27 were labeled as Hsp27. These cells were prepared for subsequent experiments.

2.5. Measurement of NO Concentration in the Medium. Cells were incubated in six-well plates and treated with $10 \mathrm{mM}$ Hcy for $24 \mathrm{~h}$. The medium was collected and the total NO production was measured using a Total Nitric Oxide Assay Kit (Beyotime Biotechnology, Shanghai, China).

2.6. Apoptosis Analysis by Flow Cytometry. After treatment, cells were detached from the culture dishes with $0.25 \%$ trypsin and were collected by centrifugation. After washing twice with ice-cold PBS, the cells were resuspended in binding buffer containing annexin V-FITC and propidium iodide (PI) according to the manufacturer's instructions. Cells were then sorted using flow cytometry.

2.7. ROS Determination by Flow Cytometry. Cells were stained with $2^{\prime}, 7^{\prime}$-dichlorofluorescein diacetate (DCFH-DA). Cells were plated in six-well plate and then treated with $10 \mathrm{mM} \mathrm{Hcy}$ for another $24 \mathrm{~h}$. After treatment, cells were incubated with $10 \mu \mathrm{M}$ DCFH-DA in the dark for $30 \mathrm{~min}$. Finally, the cells were analyzed for DCF fluorescence by flow cytometry.

2.8. MMP Determination by Flow Cytometry. Cells were plated on six-well plates and then treated with $10 \mathrm{mM} \mathrm{Hcy}$ for another $24 \mathrm{~h}$. Afterwards, the cells were incubated with Rhodamine $123(10 \mu \mathrm{M})$ at $37^{\circ} \mathrm{C}$ in the dark for $20 \mathrm{~min}$. After filtration, the samples were analyzed by flow cytometry.

2.9. qRT-PCR Analysis. Total RNA was isolated using TRIzol in a sterile, RNase-free environment. Reverse transcription was done using the RevertAid Reverse Transcriptase (Thermo Scientific); the reaction was performed at $37^{\circ} \mathrm{C}$ for $1 \mathrm{~h}$. The qRT-PCR was performed using Maxima SYBR Green qPCR Master Mix (2x) (Thermo Scientific). The reaction system contained $12.5 \mu \mathrm{L}$ of Maxima SYBR Green qPCR Master Mix $(2 \mathrm{x}), 1 \mu \mathrm{L}$ of primer, $1 \mu \mathrm{L}$ of cDNA, and $10.5 \mu \mathrm{L}$ of $\mathrm{ddH}_{2} \mathrm{O}$. We used Roche LightCycler 480 II System to perform qRTPCR. The procedure was as follows: pretreatment for 1 cycle at $95^{\circ} \mathrm{C}$ for $2 \mathrm{~min}$; initial denaturation for 1 cycle at $95^{\circ} \mathrm{C}$ for $30 \mathrm{~s} ; 40$ cycles of $95^{\circ} \mathrm{C}$ for $15 \mathrm{~s}$ and $60^{\circ} \mathrm{C}$ for $60 \mathrm{~s}$; and melt curve stage. Fold changes in target gene expression between treatments and controls were determined using the $2^{-\Delta \Delta \mathrm{Ct}}$ method, normalizing to $18 \mathrm{~S}$ RNA expression as an internal reference. All analyses were performed in six independent experiments and were performed in triplicate. The primers used were $5^{\prime}$-CGGACATCTAAGGGCATCACAG$3^{\prime}$ (sense) and $5^{\prime}$-GGACACGGACAGGATTGACA-3' (antisense) for 18S; $5^{\prime}$-ACGCAGTCCAACGAGATCA-3' (sense) and $5^{\prime}$-CTTTACTTGGCGGCAGTCTC-3' (antisense) for HSP27; $5^{\prime}$-TCTCTGCTGTTTGTGGCTTG-3' (sense) and $5^{\prime}$-GGACTGGGAGTGGGTTTCTC-3' (antisense) for ET1; $5^{\prime}$-CTCAGCCAGATGCAATCAAT-3' (sense) and $5^{\prime}$ GCTTCTTTGGGACACTTGCT-3' (antisense) for MCP1; $5^{\prime}$-AGCTTCTCCTGCTCTGCAAC-3' (sense) and $5^{\prime}$ GACAATCCCTCTCGTCCAGT-3' (antisense) for ICAM-1; and $5^{\prime}$-ACACACAGGTGGGACACAAA- $3^{\prime}$ (sense) and $5^{\prime}-$ AGGCTCCAAGGATCACGAC-3' (antisense) for VCAM-1.

2.10. Western Blot. Cellular proteins were analyzed using western blot. Cells were collected after each experiment and lysed with RIPA buffer followed by ultrasound sonication in an ice bath. The supernatant fluids were collected after centrifugation at 13,000 $\mathrm{g}$ for $5 \mathrm{~min}$. The BCA Protein Assay Kit was used to measure the protein concentrations. Gel electrophoresis was done using 10\% SDS-PAGE gel and transferred to a $0.45 \mu \mathrm{m}$ polyvinylidene fluoride membrane 


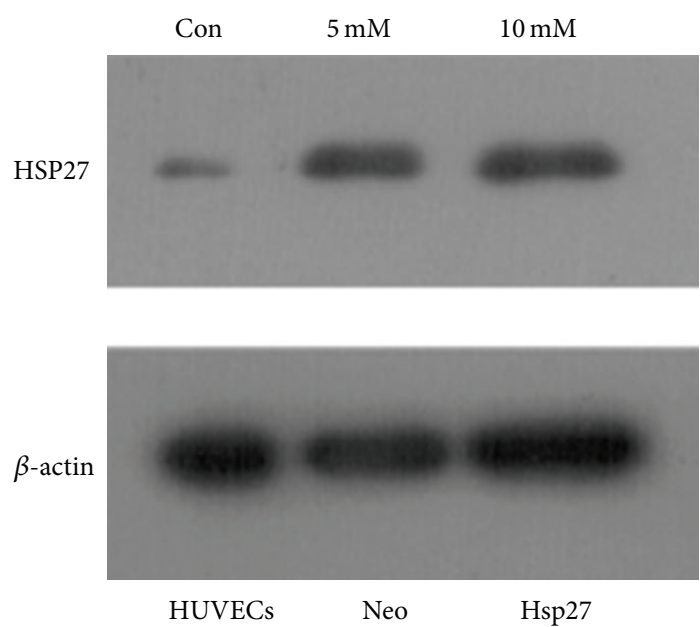

(a)

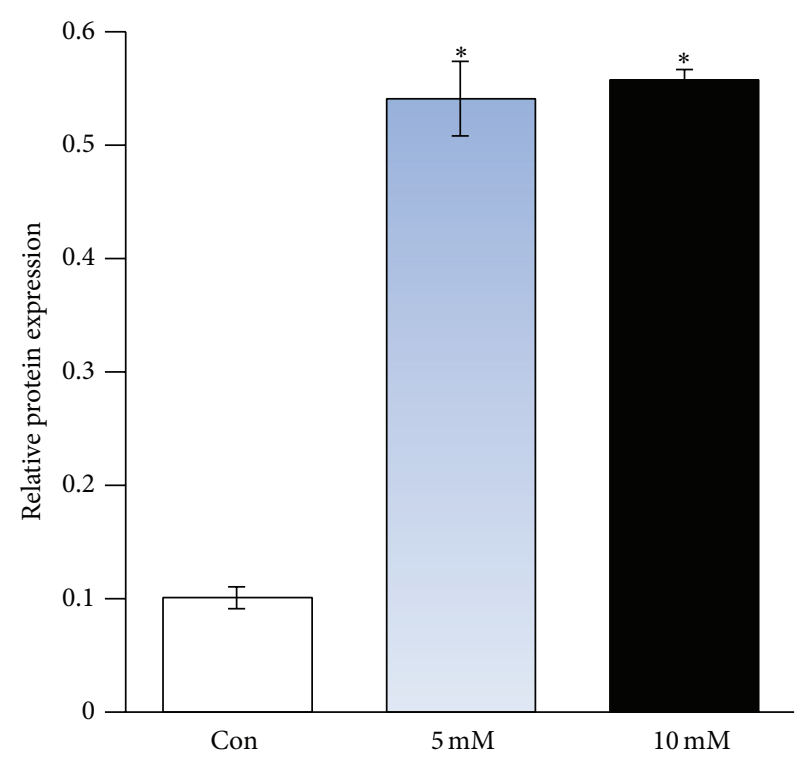

(b)

FIgURE 1: Hcy increased HSP27 expression in HUVECs. (a) Western blot analyses of the HSP27 protein levels in Hcy-treated HUVECs. Cells were treated with the indicated concentration of Hcy for $24 \mathrm{~h}$; then, they were harvested and whole-cell extracts were prepared and probed for HSP27. (b) The relative protein levels were normalized to $\beta$-actin. Data are expressed as mean \pm SD of three independent experiments. ${ }^{*} P<0.05$ compared with the control.

(Amersham Biosciences, Piscataway, NJ). The membranes were soaked in blocking buffer (5\% skimmed milk) for $1 \mathrm{~h}$. Membranes were then incubated overnight at $4^{\circ} \mathrm{C}$ with relevant antibodies, followed by horseradish peroxidaseconjugated secondary antibodies, and enhanced chemiluminescence (ECL) detection. Gel-Pro Analyzer was used to extract valuable qualitative and quantitative information from electrophoretic gels and western blot.

2.11. Statistical Analysis. Data have been expressed as mean \pm SD from at least three different experiments. Comparisons were made using a one-way ANOVA followed by Dunnett's test. Statistically significant results were indicated by $P<$ 0.05 .

\section{Results}

3.1. Hcy Increased HSP27 Expression in HUVECs. Hcy is known to induce oxidative stress, which could increase the expression of heat shock proteins such as HSP27 [25]. Therefore, we examined the effect of Hcy on the expression of HSP27 in HUVECs and found that the protein level of HSP27 was upregulated in HUVECs after treatment with Hcy (Figures 1(a) and 1(b)).

3.2. Construction of the HSP27-Overexpressing Cell Line. The functions of HSP27 have not been thoroughly investigated; accumulating reports have shown that it plays an important role in cytoprotection. To investigate the functions of HSP27 in HUVECs exposed to Hcy, stable HUVECs overexpressing HSP27 were constructed. Fluorescence microscopy images of
HUVECs, Neo, and HSP27 cells were shown in Figure 2(a). The data for mRNA and protein levels of HSP27 were confirmed by qRT-PCR and western blot (Figures 2(b), 2(c), and $2(\mathrm{~d})$ ).

\subsection{Effects of HSP27 on NO Production and Endothelial} Molecule Expression. The common feature of endothelial dysfunction is a decrease in the amount of bioavailable $\mathrm{NO}$; the production of $\mathrm{NO}$ can be a marker of endothelial dysfunction [26]. After treatment with Hcy, the level of $\mathrm{NO}$ in the culture medium of the Hsp27+Hcy group was significantly higher than that for the Neo+Hcy group (Figure 3(b)). ET-1, which is synthesized predominantly by vascular endothelial cells, is the most potent vessel constrictor and vascular modulator. The expression of ET-1 increases when endothelial dysfunction occurs [27]. Endothelial cells express adhesion and chemoattractant molecules like ICAM1, VCAM-1, and MCP-1, which are particularly implicated in vascular inflammation in atherogenic processes $[28,29]$. When endothelial dysfunction occurs, the expression of ICAM-1, VCAM-1, and MCP-1 in mRNA is upregulated [30, 31]. We found that the mRNA levels of ET-1, ICAM-1, VCAM1, and MCP-1 were higher in the Hsp27+Hcy group than the Neo+Hcy group (Figure 3(a)).

3.4. Protective Effect of HSP27 on Hcy-Induced Apoptosis. To investigate the effect of HSP27 on Hcy-induced apoptosis, we treated the cells with or without $10 \mathrm{mM} \mathrm{Hcy}$ for $24 \mathrm{~h}$ and the percentages of cells undergoing apoptosis were determined by flow cytometry analysis after staining with annexin VFITC and PI. Compared to the Neo+Hcy group, the apoptosis 

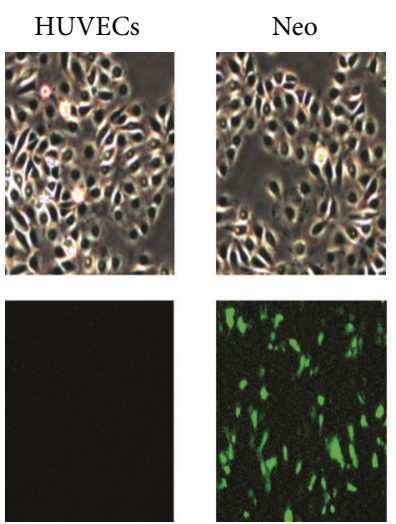

(a)
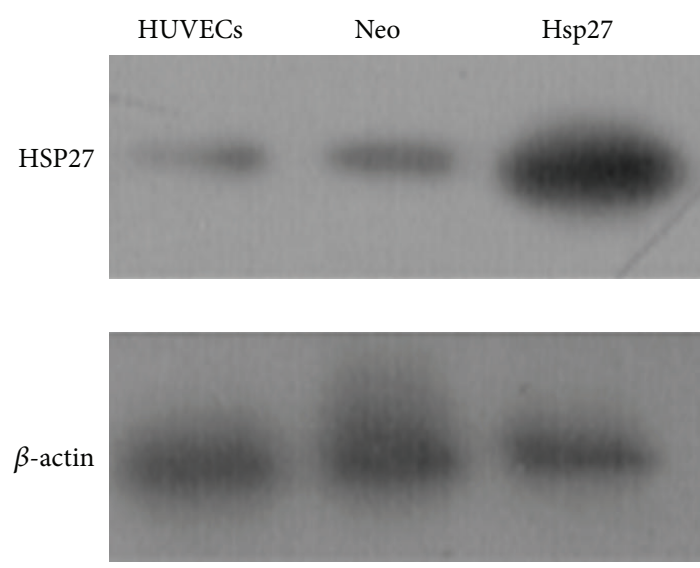

(c)

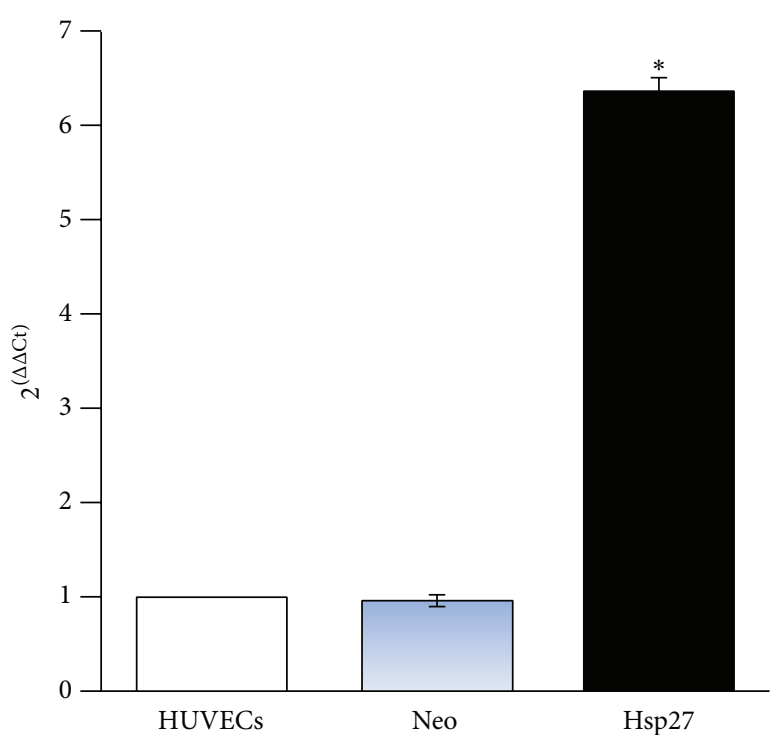

(b)

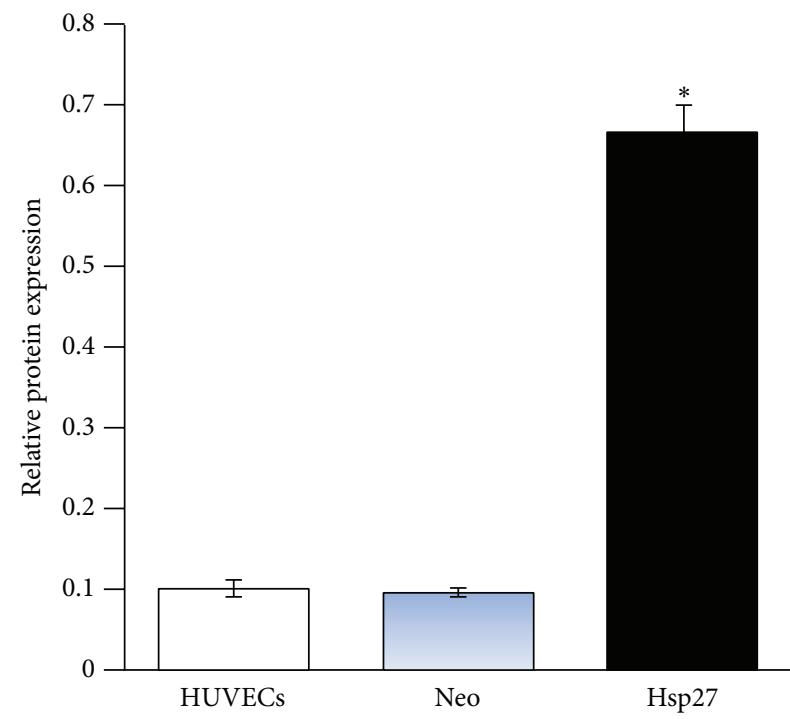

(d)

Figure 2: Stable HSP27 overexpressing HUVEC line (Hsp27). The pEX-4 or HSP27-pEX-4 plasmid was inserted in HUVECs. After 2 weeks of G418 selection, high expression of HSP27 as compared to that in parental HUVECs was detected in cells by fluorescence microscopy, qRT-PCR, and western blot analyses. (a) Images of HUVECs, Neo, and Hsp27 cells obtained by fluorescence microscopy (4x original magnification); the green fluorescence represented GFP. (b) Real-time RT-PCR analysis of HSP27 mRNA expression in cell lines. Total RNA was prepared and quantified using specific primers. (c) HSP27 protein expression by western blotting. (d) The relative protein levels were normalized to $\beta$-actin. Data are expressed as mean \pm SD of three independent experiments. ${ }^{*} P<0.05$ compared with the Neo group.

rate in the Hsp $27+\mathrm{Hcy}$ group was decreased from $88.85 \%$ to $50.93 \%$ (Figures 3(c) and 3(d)).

\subsection{HSP27 Attenuated Hcy-Mediated ROS Generation and} MMP Reduction. ROS is a mediator of intracellular signals and plays an important role in causing apoptotic cell death [32]. A significant $(P<0.05)$ increase in the intracellular ROS level was observed in Hcy-exposed Neo cells while Hsp27 cells exhibited only $\sim 17.48 \%$ increase (Figures $4(\mathrm{a})$ and 4 (b)). Meanwhile, a 77.17\% decrease of MMP was found in Hcyexposed Neo cells, while Hsp27 cells exhibited only $46.44 \%$ decrease (Figures 4(c) and 4(d)). The data showed that HSP27 had a protective effect on Hcy-induced apoptosis and MMP disruption in HUVECs.

3.6. Effects of HSP27 on the Expression of Apoptosis Regulators Induced by Hcy. The Bcl-2 protein family, a large family of apoptosis-regulating proteins, modulates the mitochondrial pathway [33]. To further characterize the function of HSP27 in Hcy-induced apoptosis, we examined the impact of HSP27 on levels of Bcl-2 family proteins in Hcy-treated HUVECs by using western blot. After treatment with Hcy, the rate of 


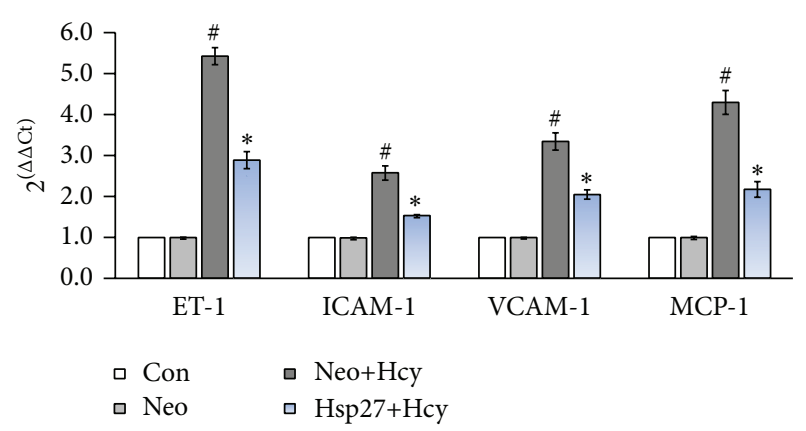

(a)

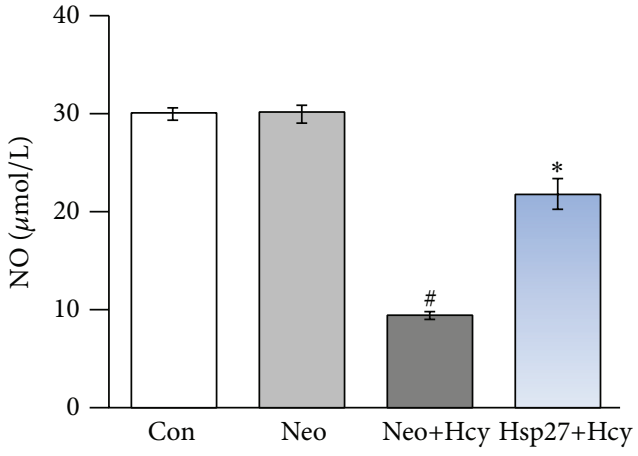

(b)

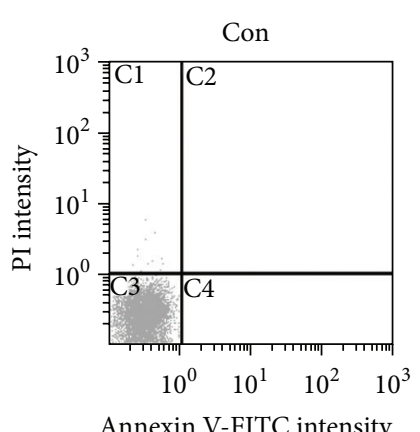

Annexin V-FITC intensity
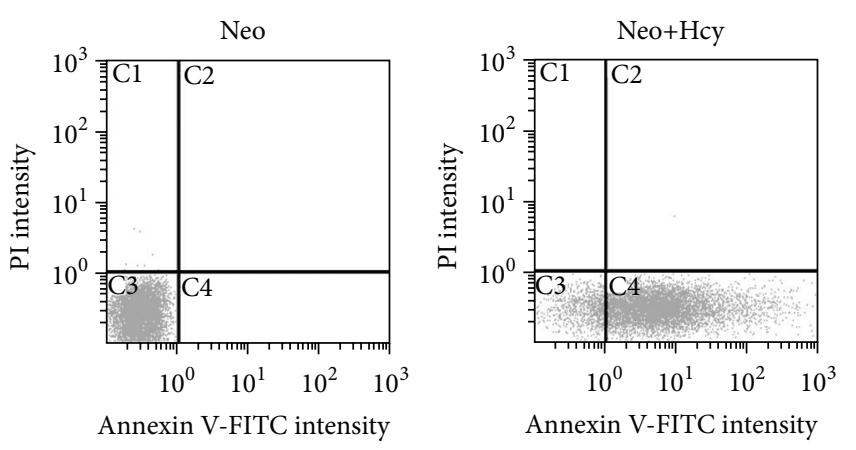

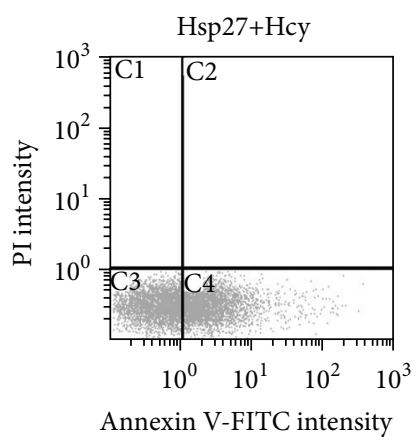

(c)

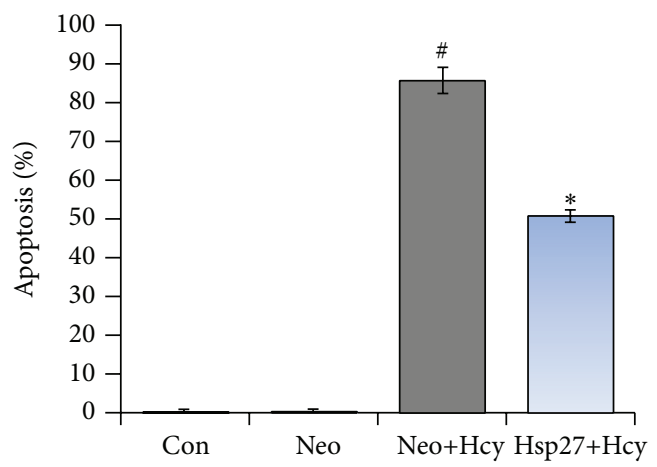

(d)

FIGURE 3: Effect of HSP27 on Hcy-induced endothelial dysfunction and apoptosis (a). Real-time RT-PCR analysis of ET-1, ICAM-1, VCAM-1, and MCP-1 mRNA expression in HUVECs. After cells were treated with $10 \mathrm{mM}$ Hcy for $24 \mathrm{~h}$ or were not treated, total RNA was prepared and quantified using specific primers. (b) HSP27 weakened the Hcy-mediated NO decrease in HUVECs. (c) and (d) Cells that were treated with $10 \mathrm{mM} \mathrm{Hcy}$ or were not treated were analyzed after $24 \mathrm{~h}$ by flow cytometry. The number represents the percentages of apoptotic cells in each condition. Data are expressed as mean \pm SD of three independent experiments. Columns not sharing the same superscript letter differ significantly: ${ }^{*} P<0.05$ compared with the Neo+Hcy group; ${ }^{\#} P<0.05$ compared with the Neo group.

Bcl-2/Bax was increased in the Hsp27+Hcy group compared to Neo+Hcy group (Figures 5(a) and 5(c)). In addition, caspase-3 cleavage and PARP cleavage both decreased in the $\mathrm{Hsp} 27+\mathrm{Hcy}$ group compared to the $\mathrm{Neo}+\mathrm{Hcy}$ group (Figures 5(b) and 5(c)).

\section{Discussion}

Increased plasma level of Hcy is an independent risk factor for the development of atherosclerosis [3-5]. Evidence shows that the elevation of plasma Hcy can lead to endothelial dysfunction [34, 35]. Hsp27, as a cytoprotector, has been reported to be a potent antiapoptotic molecule [11, 36, 37]. In order to investigate the role of HSP27 in endothelial cells, we tested the expression of HSP27 in Hcy-treated HUVECs. We found that Hcy treatment promoted HSP27 expression in HUVECs, while overexpression of HSP27 decreased Hcyinduced endothelial apoptosis. This may indicate that HSP27 regulated cell apoptosis in a negative feedback form.

$\mathrm{NO}$ is a crucial mediator in endothelial vasodilator function. Abnormal NO production in the vascular endothelium results in endothelial dysfunction, which is a prelude to 


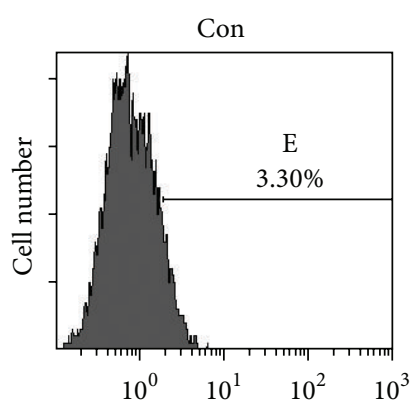

DCF fluorescence

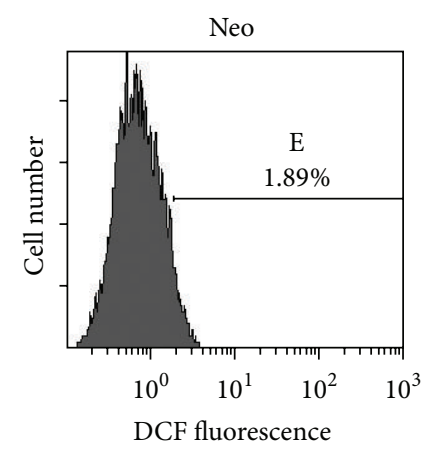

DCF fluorescence
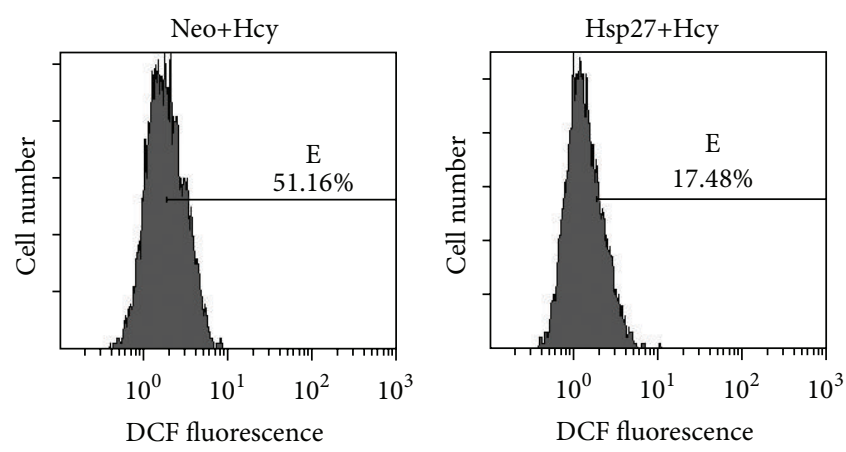

(a)

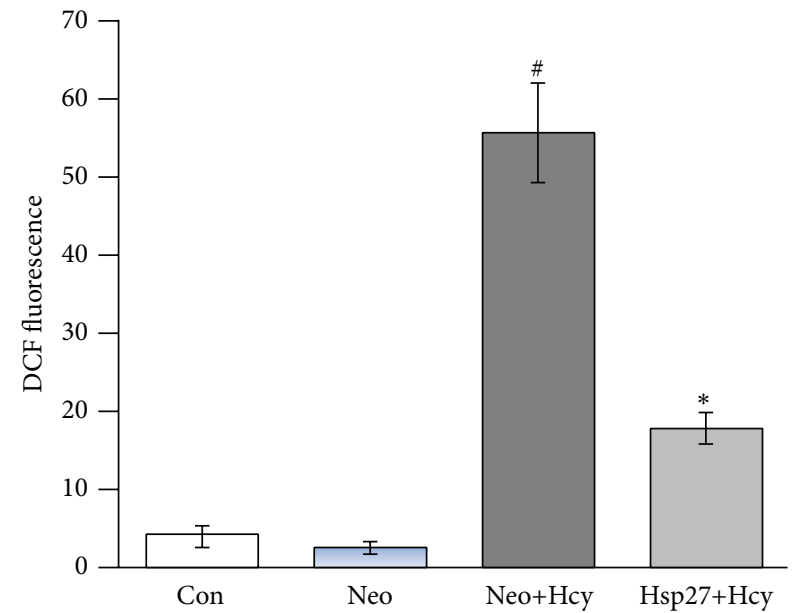

(b)

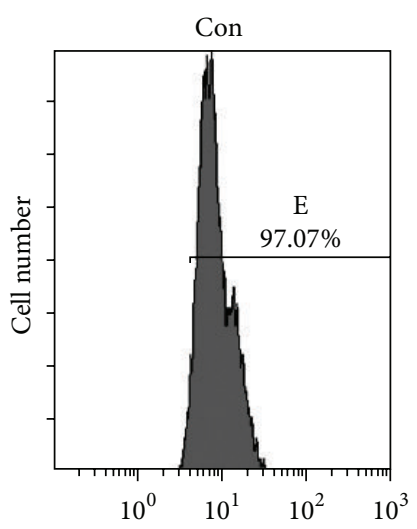

Rh123 fluorescence

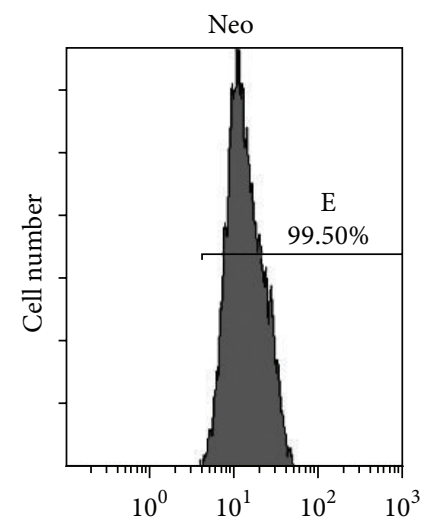

Rh123 fluorescence

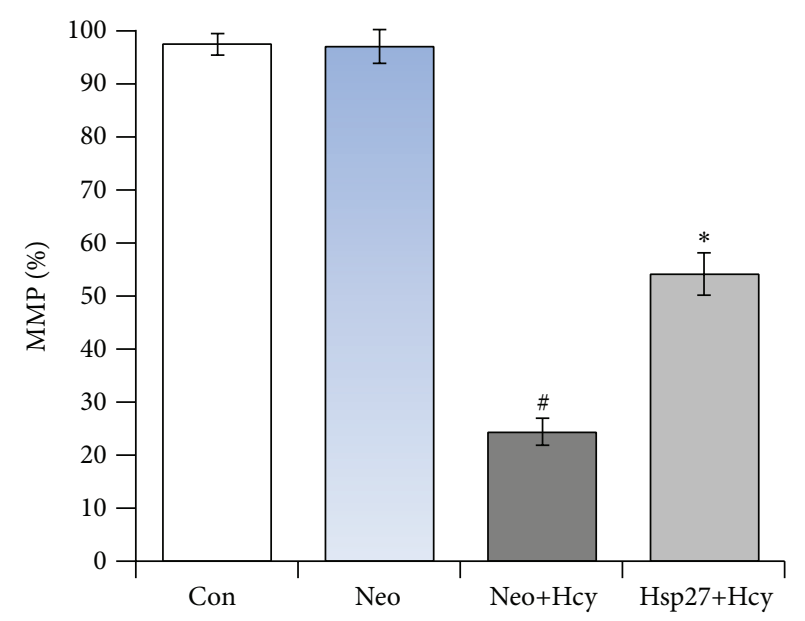

(c)
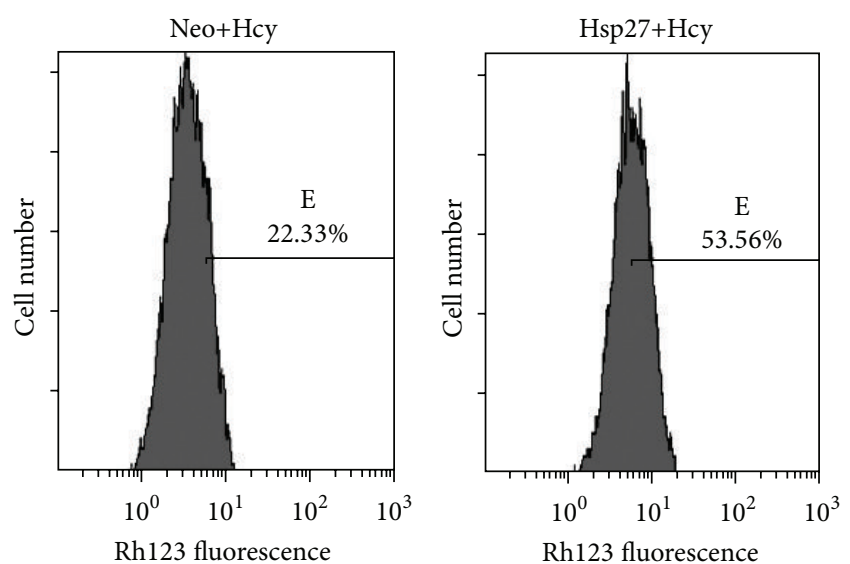

(d)

FIgURE 4: HSP27 resisted Hcy-induced elevation in ROS and MMP depletion. (a) ROS in cells that were treated with $10 \mathrm{mM}$ Hcy or were not treated were analyzed after $24 \mathrm{~h}$ by flow cytometry. (b) and (c) Data are expressed as mean \pm SD of three independent experiments. Columns not sharing the same superscript letter differ significantly: ${ }^{*} P<0.05$ compared with Neo+Hcy group; ${ }^{\#} P<0.05$ compared with the Neo group. (d) Cells were treated with $10 \mathrm{mM} \mathrm{Hcy}$ for $24 \mathrm{~h}$ or were not treated; then, they were collected and stained with Rh123 for flow cytometric analysis. The values indicated the percentage of Rh123 fluorescence on cells after the treatment.

atherosclerosis [38]. In the vascular system, the bioavailability of NO can be impaired by various mechanisms such as decrease in NO production due to eNOS and enhanced NO breakdown during oxidative stress. NO reacts with superoxide to form peroxynitrite. Peroxynitrite changes the redox state of the vessel wall, which might stimulate increased expression of VCAM-1, ICAM-1, E-selectin, and MCP-1 in the endothelial cells [26]. In this study, Hcy inhibited NO synthesis while HSP27 rescued NO synthesis, thereby improving endothelial function. ET-1, a potent vasoconstrictor, is primarily produced by vascular endothelial cells. ET1 and $\mathrm{NO}$ are natural counterparts in vascular function. 

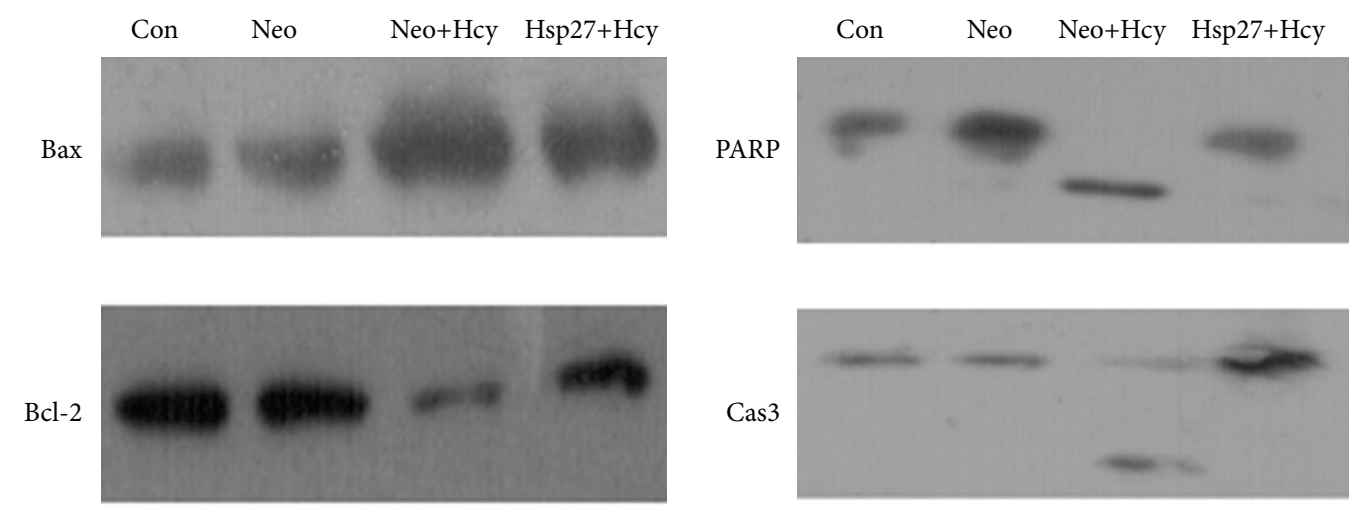

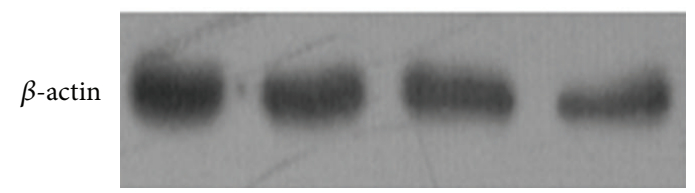

(a)

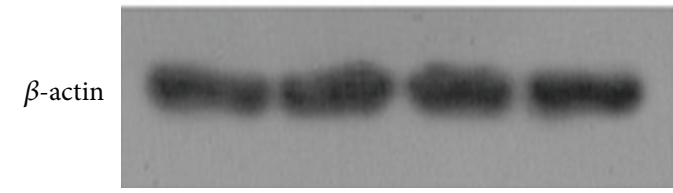

(b)

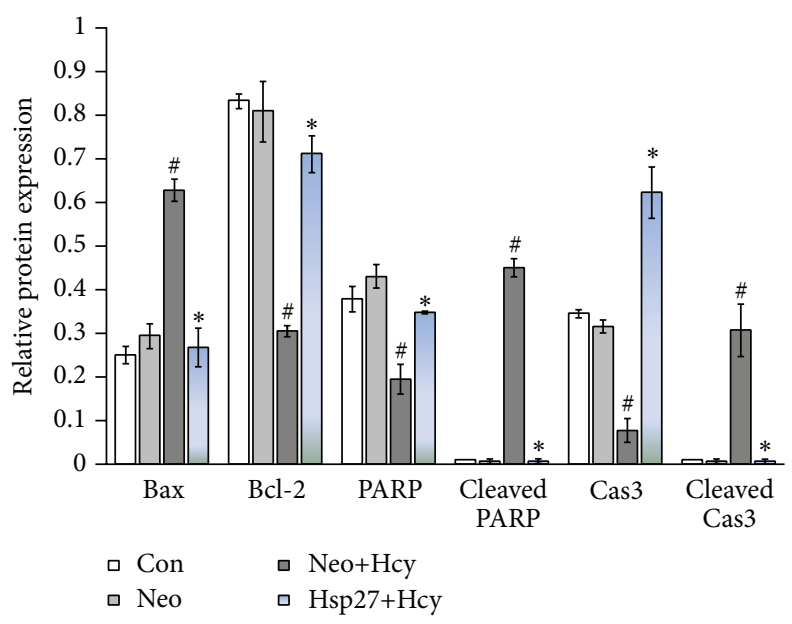

(c)

FIGURE 5: HSP27 attenuated the effect of Hcy on the expression of apoptosis regulators. Cells were treated with $10 \mathrm{mM} \mathrm{Hcy} \mathrm{for} 24 \mathrm{~h}$ or were not treated. (a) and (b) Whole-cell extracts were prepared and probed for Bcl-2, Bax, caspase-3, and PARP by western blot analysis and quantified by the Gel-Pro Analyzer software. (c) The protein levels were normalized relative to those of $\beta$-actin, which was used as a loading control. Data are expressed as mean \pm SD of three independent experiments. Columns not sharing the same superscript letter differ significantly: ${ }^{*} P<0.05$ compared with the Neo+Hcy group; ${ }^{\#} P<0.05$ compared with the Neo group.

ET-1 modulates endothelial function and is upregulated after endothelial dysfunction [27, 39]. Endothelial cells also express adhesion and chemoattractant molecules such as MCP-1, VCAM-1, and ICAM-1, which recruit inflammatory monocytes into the vascular wall and initiate atherosclerosis $[29,40]$. When endothelial dysfunction occurs, the mRNA and protein expression of ICAM-1, VCAM-1, and MCP-1 increases $[26,41,42]$. The results showed that Hcy increased the mRNA levels of ET-1, ICAM-1, VCAM-1, and MCP1 , and these effects could be attenuated further by HSP27. These results demonstrated that HSP27 rescued endothelial dysfunction after exposure to Hcy.

It has been reported that oxidative stress plays an important role in the pathogenesis of endothelial dysfunction and atherosclerosis [43, 44]. Elevated amounts of intracellular ROS can induce oxidative stress, loss of cell function, and cell apoptosis [45]. It has been shown that MMP loss, an early event, is directly associated with apoptosis [46]. As MMP decreases, the mitochondrial permeability transit pores (PTPs) open and cytochrome $\mathrm{c}$ and other proapoptotic molecules are released from the intermembrane space to the cytosol. In this study, HUVECs overexpressing HSP27 resisted intracellular ROS level elevation and MMP depletion after exposure to Hcy. Considering these results, it is evident that HSP27 could inhibit Hcy-induced endothelial apoptosis by decreasing intracellular ROS level and increasing MMP.

Bcl-2 family proteins, which include antiapoptotic proteins and proapoptotic proteins such as $\mathrm{Bcl}-2$ and Bax, govern 
the mitochondria-dependent apoptosis pathway [33]. The results of this study showed that the expressions of Bax were increased and Bcl-2 was decreased in Hcy-treated cells, while HSP27 weakened this response. Caspase-3 has been considered as a central component of the proteolytic cascade during apoptosis and can cleave some nuclear proteins such as PARP, thereby leading to a typical apoptotic DNA fragmentation [47]. In this study, HSP27 decreased the expression of cleaved PARP and cleaved caspase- 3 after treatment with Hcy. These results showed that HSP27 inhibited Hcy-induced endothelial apoptosis by modulating the mitochondrial caspasedependent apoptotic pathway.

In conclusion, HSP27 protected against Hcy-induced endothelial apoptosis, possibly by inducing cellular ROS production and inhibiting the mitochondrial caspase-dependent apoptotic pathway. The effects of HSP27 on endothelial molecule expression and NO production represent a novel mechanism by which HSP27 modulates vascular homeostasis and may serve as a new approach for prevention of atherosclerosis. However, further research on the important role of HSP27 in protecting endothelial cells from apoptosis in vivo is warranted.

\section{Competing Interests}

The authors declare that there are no conflicts of interest regarding the publication of this paper. They also confirm that those findings mentioned above do not lead to any conflicts of interest regarding the publication of this paper.

\section{Acknowledgments}

This work was supported by Wu Jieping medical Foundation (no. 320.6750.12265) and the Natural Science Foundation of China (no. 81570520).

\section{References}

[1] C. K. Glass and J. L. Witztum, "Atherosclerosis. The road ahead," Cell, vol. 104, no. 4, pp. 503-516, 2001.

[2] G. K. Hansson, "Mechanisms of disease: inflammation, atherosclerosis, and coronary artery disease," The New England Journal of Medicine, vol. 352, no. 16, pp. 1626-1695, 2005.

[3] J.-C. Guilland, A. Favier, G. Potier de Courcy, P. Galan, and S. Hercberg, "Hyperhomocysteinemia: an independent risk factor or a simple marker of vascular disease?. 1. Basic data," Pathologie Biologie, vol. 51, no. 2, pp. 101-110, 2003.

[4] A. B. Lawrence de Koning, G. H. Werstuck, J. Zhou, and R. C. Austin, "Hyperhomocysteinemia and its role in the development of atherosclerosis," Clinical Biochemistry, vol. 36, no. 6, pp. 431-441, 2003.

[5] R. Clarke, L. Daly, K. Robinson et al., "Hyperhomocysteinemia: an independent risk factor for vascular disease," The New England Journal of Medicine, vol. 324, no. 17, pp. 1149-1155, 1991.

[6] B. Horvath, L. Szapary, L. Debreceni et al., "Effect of sclerovit on endothelial dysfunction, hemorheological parameters, platelet aggregation, plasma concentration of homocysteine and progression of atherosclerosis in patients with vascular diseases," Clinical Hemorheology and Microcirculation, vol. 42, no. 1, pp. 19-28, 2009.
[7] A. Briasoulis, D. Tousoulis, E. S. Androulakis, N. Papageorgiou, G. Latsios, and C. Stefanadis, "Endothelial dysfunction and atherosclerosis: focus on novel therapeutic approaches," Recent Patents on Cardiovascular Drug Discovery, vol. 7, no. 1, pp. 21-32, 2012.

[8] J. Davignon and P. Ganz, "Role of endothelial dysfunction in atherosclerosis," Circulation, vol. 109, no. 23, pp. III27-III32, 2004.

[9] B.-J. Martin and T. J. Anderson, "Risk prediction in cardiovascular disease: the prognostic significance of endothelial dysfunction," The Canadian Journal of Cardiology, vol. 25, pp. 15A-20A, 2009.

[10] F. Chen, P. Eriksson, T. Kimura, I. Herzfeld, and G. Valen, "Apoptosis and angiogenesis are induced in the unstable coronary atherosclerotic plaque," Coronary Artery Disease, vol. 16, no. 3, pp. 191-197, 2005.

[11] C. G. Concannon, A. M. Gorman, and A. Samali, "On the role of Hsp27 in regulating apoptosis," Apoptosis, vol. 8, no. 1, pp. 61-70, 2003.

[12] S. Salari, T. Seibert, Y.-X. Chen et al., "Extracellular HSP27 acts as a signaling molecule to activate NF- $\kappa \mathrm{B}$ in macrophages," Cell Stress \& Chaperones, vol. 18, no. 1, pp. 53-63, 2013.

[13] H.-F. Chen, L.-D. Xie, and C.-S. Xu, "Role of heat shock protein 27 phosphorylation in migration of vascular smooth muscle cells," Molecular and Cellular Biochemistry, vol. 327, no. 1-2, pp. $1-6,2009$.

[14] J. L. Martin-Ventura, M. C. Duran, L. M. Blanco-Colio et al., "Identification by a differential proteomic approach of heat shock protein 27 as a potential marker of atherosclerosis," Circulation, vol. 110, no. 15, pp. 2216-2219, 2004.

[15] C. A. Efthymiou, M. M. Mocanu, J. de Belleroche, D. J. Wells, D. S. Latchmann, and D. M. Yellon, "Heat shock protein 27 protects the heart against myocardial infarction," Basic Research in Cardiology, vol. 99, no. 6, pp. 392-394, 2004.

[16] H. Miller, S. Poon, B. Hibbert, K. Rayner, Y.-X. Chen, and E. R. O'Brien, "Modulation of estrogen signaling by the novel interaction of heat shock protein 27 , a biomarker for atherosclerosis, and estrogen receptor beta: mechanistic insight into the vascular effects of estrogens," Arteriosclerosis, Thrombosis, and Vascular Biology, vol. 25, no. 3, pp. e10-e14, 2005.

[17] A. Pandey, S. Saini, R. Khatoon, D. Sharma, G. Narayan, and D. Kar Chowdhuri, "Overexpression of hsp27 rescued neuronal cell death and reduction in life- and health-span in drosophila melanogaster against prolonged exposure to dichlorvos," Molecular Neurobiology, 2015.

[18] B. Zhang, H. S. Zhou, Q. Cheng, L. Lei, and B. Hu, "Overexpression of HSP27 in cultured human aortic smooth muscular cells reduces apoptosis induced by low-frequency and low-energy ultrasound by inhibition of an intrinsic pathway," Genetics and Molecular Research, vol. 12, no. 4, pp. 6588-6601, 2013.

[19] L. Korngut, C. H. E. Ma, J. A. Martinez et al., "Overexpression of human HSP27 protects sensory neurons from diabetes," Neurobiology of Disease, vol. 47, no. 3, pp. 436-443, 2012.

[20] C. Kubisch, M. J. Dimagno, A. B. Tietz et al., "Overexpression of heat shock protein Hsp27 protects against cerulein-induced pancreatitis," Gastroenterology, vol. 127, no. 1, pp. 275-286, 2004.

[21] A. Wyttenbach, O. Sauvageot, J. Carmichael, C. Diaz-Latoud, A.-P. Arrigo, and D. C. Rubinsztein, "Heat shock protein 27 prevents cellular polyglutamine toxicity and suppresses the increase of reactive oxygen species caused by huntingtin," Human Molecular Genetics, vol. 11, no. 9, pp. 1137-1151, 2002. 
[22] A.-P. Arrigo, S. Virot, S. Chaufour, W. Firdaus, C. Kretz-Remy, and C. Diaz-Latoud, "Hsp27 consolidates intracellular redox homeostasis by upholding glutathione in its reduced form and by decreasing iron intracellular levels," Antioxidants \& Redox Signaling, vol. 7, no. 3-4, pp. 414-424, 2005.

[23] P. Pandey, R. Farber, A. Nakazawa et al., "Hsp27 functions as a negative regulator of cytochrome c-dependent activation of procaspase-3," Oncogene, vol. 19, no. 16, pp. 1975-1981, 2000.

[24] C. G. Concannon, S. Orrenius, and A. Samali, "Hsp27 inhibits cytochrome c-mediated caspase activation by sequestering both pro-caspase-3 and cytochrome c," Gene Expression, vol. 9, no. 45, pp. 195-201, 2001.

[25] N. R. Madamanchi, S. Li, C. Patterson, and M. S. Runge, "Reactive oxygen species regulate heat-shock protein 70 via the JAK/STAT pathway," Arteriosclerosis, Thrombosis, and Vascular Biology, vol. 21, no. 3, pp. 321-326, 2001.

[26] P. L. Huang, "Endothelial nitric oxide synthase and endothelial dysfunction," Current Hypertension Reports, vol. 5, no. 6, pp. 473-480, 2003.

[27] S. L. Bourque, S. T. Davidge, and M. A. Adams, "The interaction between endothelin-1 and nitric oxide in the vasculature: new perspectives," American Journal of Physiology-Regulatory Integrative and Comparative Physiology, vol. 300, no. 6, pp. R1288-R1295, 2011.

[28] M. Takahashi, "Inflammatory cytokines in the pathogenesis of atherosclerosis," Nihon Rinsho. Japanese Journal of Clinical Medicine, vol. 69, no. 1, pp. 30-33, 2011.

[29] P. J. Little, A. Chait, and A. Bobik, "Cellular and cytokinebased inflammatory processes as novel therapeutic targets for the prevention and treatment of atherosclerosis," Pharmacology and Therapeutics, vol. 131, no. 3, pp. 255-268, 2011.

[30] H.-P. Zhang, F.-L. Zheng, J.-H. Zhao, D.-X. Guo, and X.L. Chen, "Genistein inhibits ox-ldl-induced vcam-1, icam-1 and mcp-1 expression of huvecs through heme oxygenase-1," Archives of Medical Research, vol. 44, no. 1, pp. 13-20, 2013.

[31] Y. Lu, X. Zhu, G.-X. Liang et al., "Apelin-APJ induces ICAM-1, VCAM- 1 and MCP-1 expression via NF- $\kappa$ B/JNK signal pathway in human umbilical vein endothelial cells," Amino Acids, vol. 43, no. 5, pp. 2125-2136, 2012.

[32] S. Clutton, "The importance of oxidative stress in apoptosis," British Medical Bulletin, vol. 53, no. 3, pp. 662-668, 1997.

[33] A. Frenzel, F. Grespi, W. Chmelewskij, and A. Villunger, "Bcl2 family proteins in carcinogenesis and the treatment of cancer," Apoptosis, vol. 14, no. 4, pp. 584-596, 2009.

[34] F. M. Faraci and S. R. Lentz, "Hyperhomocysteinemia, oxidative stress, and cerebral vascular dysfunction," Stroke, vol. 35, no. 2, pp. 345-347, 2004.

[35] K. S. Woo, M. Qiao, P. Chook et al., "Homocysteine, endothelial dysfunction, and coronary artery disease: emerging strategy for secondary prevention," Journal of Cardiac Surgery, vol. 17, no. 5, pp. 432-435, 2002.

[36] C. Garrido, E. Schmitt, C. Candé, N. Vahsen, A. Parcellier, and G. Kroemer, "HSP27 and HSP70: potentially oncogenic apoptosis inhibitors," Cell Cycle, vol. 2, no. 6, pp. 579-584, 2003.

[37] M. de Graauw, I. Tijdens, R. Cramer, S. Corless, J. F. Timms, and B. van de Water, "Heat shock protein 27 is the major differentially phosphorylated protein involved in renal epithelial cellular stress response and controls focal adhesion organization and apoptosis," The Journal of Biological Chemistry, vol. 280, no. 33, pp. 29885-29898, 2005.
[38] Y. Higashi, K. Noma, M. Yoshizumi, and Y. Kihara, "Endothelial function and oxidative stress in cardiovascular diseases," Circulation Journal, vol. 73, no. 3, pp. 411-418, 2009.

[39] A. Lerman, D. R. Holmes Jr., M. R. Bell, K. N. Garratt, R. A. Nishimura, and J. C. Burnett Jr., "Endothelin in coronary endothelial dysfunction and early atherosclerosis in humans," Circulation, vol. 92, no. 9, pp. 2426-2431, 1995.

[40] E. Zakynthinos and N. Pappa, "Inflammatory biomarkers in coronary artery disease," Journal of Cardiology, vol. 53, no. 3, pp. 317-333, 2009.

[41] S. A. Doggrell, "The endothelin system and its role in acute myocardial infarction," Expert Opinion on Therapeutic Targets, vol. 8, no. 3, pp. 191-201, 2004.

[42] R. H. Eibl and M. Benoit, "Molecular resolution of cell adhesion forces," IEE Proceedings Nanobiotechnology, vol. 151, no. 3, pp. 128-132, 2004.

[43] H. Cai and D. G. Harrison, "Endothelial dysfunction in cardiovascular diseases: the role of oxidant stress," Circulation Research, vol. 87, no. 10, pp. 840-844, 2000.

[44] F. de Nigris, A. Lerman, L. J. Ignarro et al., "Oxidation-sensitive mechanisms, vascular apoptosis and atherosclerosis," Trends in Molecular Medicine, vol. 9, no. 8, pp. 351-359, 2003.

[45] A. F. G. Slater, C. Stefan, I. Nobel, D. J. van den Dobbelsteen, and S. Orrenius, "Signalling mechanisms and oxidative stress in apoptosis," Toxicology Letters, vol. 82-83, pp. 149-153, 1995.

[46] J. J. Lemasters, "V. Necrapoptosis and the mitochondrial permeability transition: shared pathways to necrosis and apoptosis," The American Journal of Physiology, vol. 276, no. 1, pp. G1-G6, 1999.

[47] A. G. Porter and R. U. Jänicke, "Emerging roles of caspase-3 in apoptosis," Cell Death and Differentiation, vol. 6, no. 2, pp. 99104, 1999. 


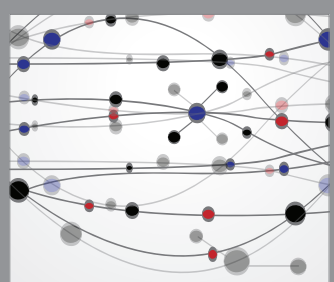

The Scientific World Journal
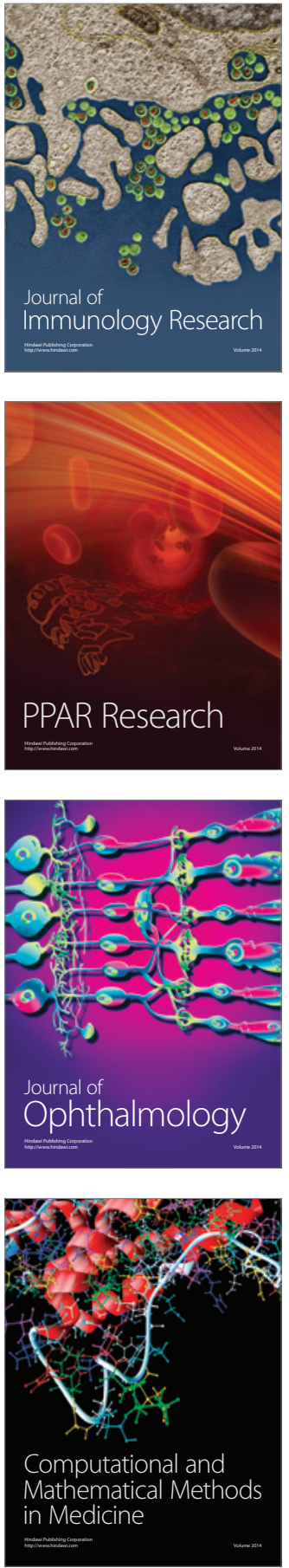

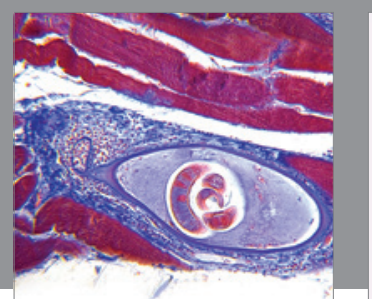

Gastroenterology Research and Practice

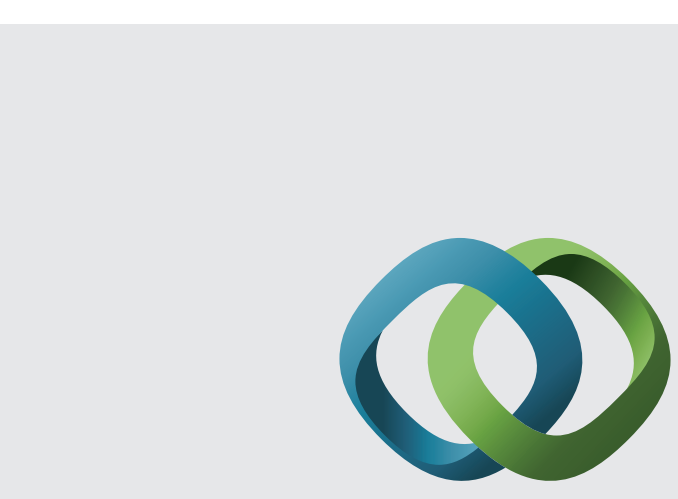

\section{Hindawi}

Submit your manuscripts at

http://www.hindawi.com
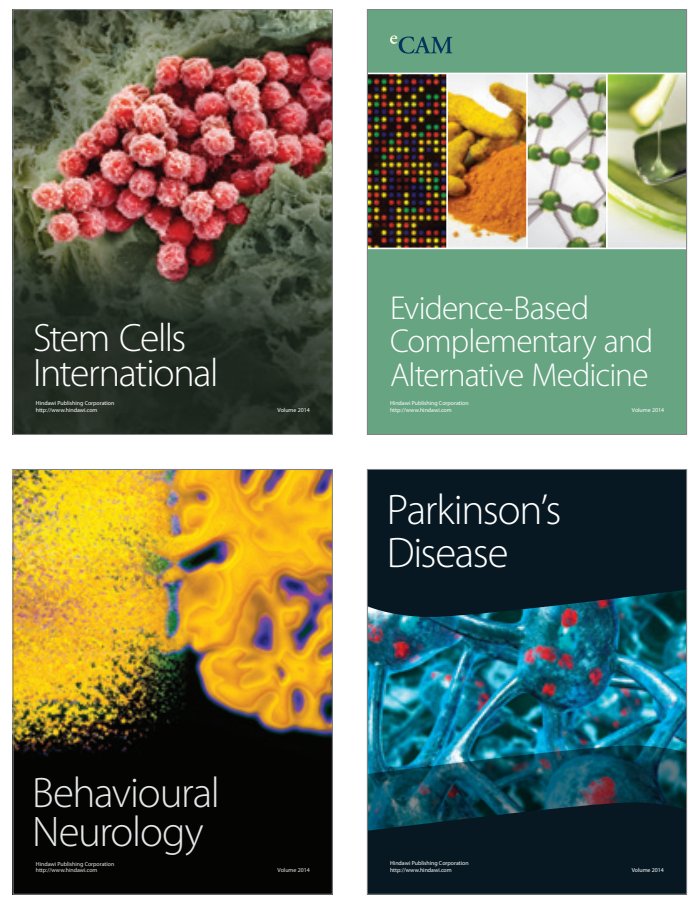
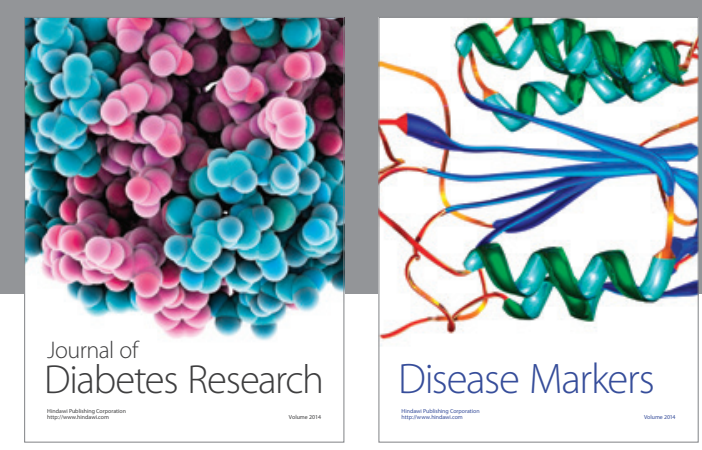

Disease Markers
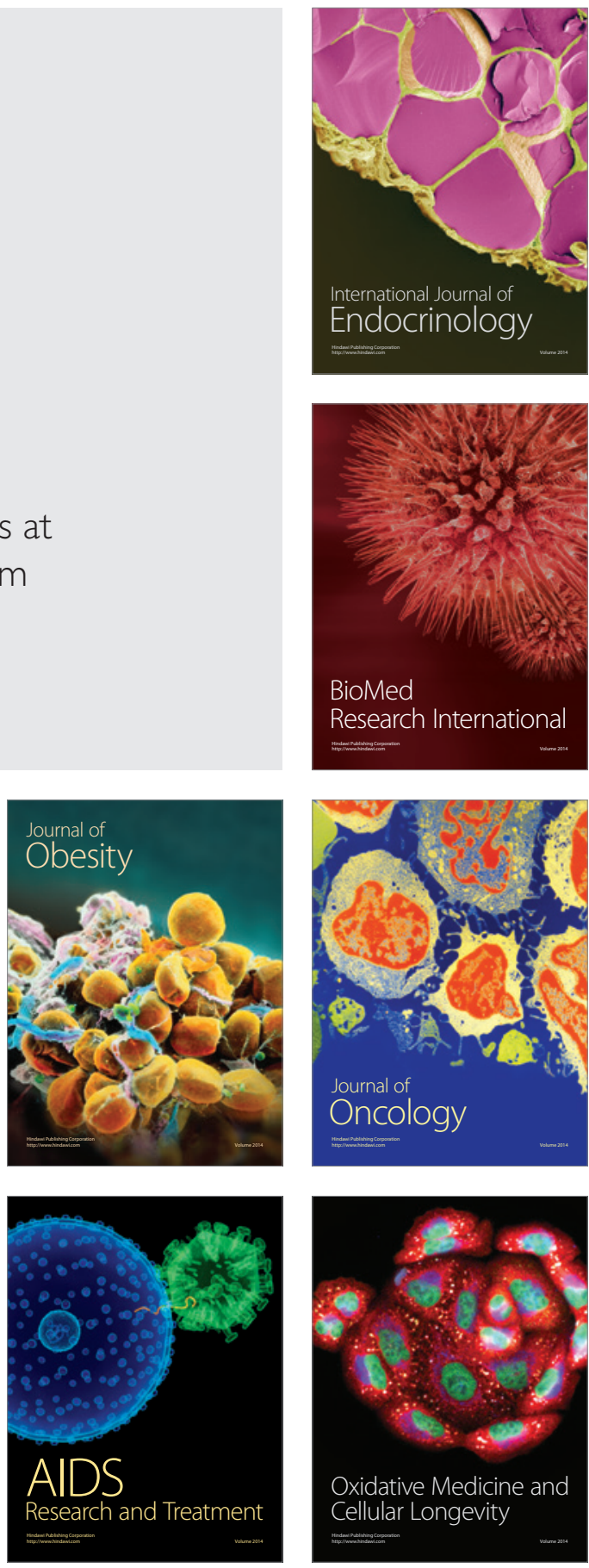